ISSN: $1231-4005$

e-ISSN: $2354-0133$

ICID: 1134116

DOI: $10.5604 / 12314005.1134116$

\title{
WEAR CUMULATING PROCESS FOR FRICTION PAIR IMPLEMENTED BY FIRST ORDER RECURRENCES
}

\author{
Krzysztof Wierzcholski \\ Technical University of Koszalin \\ Institute of Technology and Education \\ Śniadeckich Street 2, 75-453 Koszalin, Poland \\ tel.: +48943478344, fax: +48943426753 \\ e-mail: krzysztof.wierzcholski@wp.pl
}

\begin{abstract}
Wear determination engages a major part of our efforts in considered numerous tribology problems including animal teeth and joints, human joints, cams, piston, rings, tires, brakes, dirt seals, belts, shoes, fabrics, electrical contacts, tape and CD reader heads and others. At times it seems after numerous observation, that the rate of progress in the knowledge of wear is very slow. Modern design activities are mostly concern in the wear cumulative values determination rather than the wear values determination in successive time units of friction nods during the operation time. Therefore, presented paper determines in analytical way the cumulative wear values of friction nods after specified finite and infinite units of operation time. Therefore are considered the convergences and divergences of wear value cumulative processes for various friction nods. The kind of friction nods depends on type of wear process propagation. Such propagation is described in this paper by the recurrence first order equation. Mentioned equation is formulated with constant or variable parameters. This parameters are determined by virtue of experimental measurements and depend on: bearing materials properties, environmental conditions, roughness of cooperating surfaces, vibration effects, electrical conductivity of materials, hardness of materials, deformations mode of cooperating surfaces, temperature, air humidity, kind of the friction, sliding velocity, loading of the friction nod and others. The range of wearing influence components and devices is endless. Therefore, this paper presents the methods of solutions of numerous specific class of ordinary non-homogeneous recurrence equations of first order with variable coefficients occurring in hydrodynamic theory of bearing especially micro-bearing wear problems. The obtained solutions are examined by means of the UOS operator. The examples presented in this paper determine the abrasive wear cumulative values of micro-bearing system after the indicated time units of operating time. Moreover, after infinite time units of micro-bearing operation are considered the convergences and divergences of the obtained sums of cumulative wear process.
\end{abstract}

Keywords: cumulative wear values, operation after finite and infinite time units, first order recurrence implementation, variable coefficients after experiments

\section{Cumulative wear values during the micro-bearing operation time}

Up to now the practical wear cumulative equations are to be very complicated and not exactly determined [1-5]. The designers need a lot useful methods of describe the cumulative wear process for micro bearing during the operation time [5-7]. However many efforts in this direction are mostly evolutionary rather than revolutionary because desired solutions may include choice of bearing materials, choice of lubricants, choice of contact conditions, choice of the manner of operation of the mechanical systems, etc. [8-10]. The presented paper shows the new wear cumulative calculation method and new solutions of cumulative abrasive wear values of two cooperating surfaces in micro and nano scale. The functions of cumulative wear results and its limits of two cooperating HDD micro-bearing surfaces during the operation time, will be described by the class of non-homogeneous recurrent first order equation with variable coefficient and variable free term in general $[13,14]$. Achievements of presented problem can be possible on the basis of a new obtained AFM measurements of micro-bearing wear during the first period of operation time for various micro-bearing surfaces and for various mechanical system as well various operation mode $[3,11,12,15]$. 


\section{General recurrent implementation for cumulative wear}

Equation of cumulative wear process is defined in following recurrent form [14]:

$$
\mathrm{f}_{\mathrm{n}+1}=A_{n} \mathrm{f}_{\mathrm{n}}+B_{\mathrm{n}}, \quad f_{1} \equiv W, \quad F_{N} \equiv f_{1}+f_{2}+\ldots+f_{N} \quad \text { for } \quad \mathrm{n}=1,2,3, \ldots, \mathrm{N} . ; N=1,2, \ldots, \infty .
$$

Discrete wear values increases $\mathrm{f}_{\mathrm{n}+1}$ in $\mathrm{mm}^{3}$ or $\mu \mathrm{m}^{3}$ of HDD micro-bearing journal and sleeve as well the wear values sequence $\left\{\mathrm{f}_{\mathrm{n}}\right\}$ are defined for particular time units of operation $\mathrm{n}=1,2,3, \ldots, \mathrm{N}$ where $\mathrm{N}$ denotes the chosen time units of operation which can be finite or infinite number. Symbol $\mathrm{F}_{\mathrm{N}}$ describes the cumulative wear value after $\mathrm{N}$ time units of micro-bearing operation time. After experimental AFM measurements follows, that discrete wear values $\mathrm{f}_{\mathrm{n}+1}$ of the sequence $\left\{\mathrm{f}_{\mathrm{n}}\right\}$ for $\mathrm{n}=1,2,3, \ldots$ i.e. the wear values increases in $\mathrm{mm}^{3}$ or $\mu \mathrm{m}^{3}$ of HDD micro-bearing journal and sleeve equal to sum $\left(\mathrm{A}_{\mathrm{n}} \mathrm{f}_{\mathrm{n}}+\mathrm{B}_{\mathrm{n}}\right.$ ) of wear in foregoing successive time units (may be months) where addends are multiplied by dimensionless variable stochastic wear coefficient $A_{n}$ plus some dimensional, variable stochastic coefficient describing values function of materials $B_{n}$. Variable coefficients $A_{n}, B_{n}$ depend on the working parameters obtained in experiments. These parameters are as follows: bearing materials physical and chemical properties, enviromental conditions, micro and nano-roughness of cooperating surfaces, vibration effects including amplitudes and frequencies, electrical properties of materials including permeability and conductivity, hardness of materials, deformations mode of cooperating surfaces, thermal conductivity of materials, air humidity, kind of the friction, sliding velocity, stationary or unsteady loading of the friction nod and others. To solve mentioned problem of wear cumulative function it is necessary to add the boundary conditions. Hence, to the definition (1) we add condition that in first time unit, the wear obtained from experiments attains dimensional values W expressed in $\mathrm{mm}^{3}$ or $\mu^{3}$. Taking into account the physical correctness, we assume dimensionless values $\mathrm{A}_{\mathrm{n}}$ [1], and dimensional value $\mathrm{B}_{\mathrm{n}}, \mathrm{W}, \mathrm{f}_{\mathrm{n}}, \mathrm{f}_{\mathrm{N}}$ in $\mathrm{mm}^{3}$ or $\mu \mathrm{m}^{3}$. Symbols $\mathrm{n}, \mathrm{N}$ denote only natural numbers.

\section{First case of wear cumulative process identifications}

In this intersection in first case we assume variable dimensionless coefficients $A_{n} \neq 0$, and dimensional free term $B_{n}$ equal to zero. Hence, we consider the following simple particular case of wear recurrence and cumulative wear equation $[13,14]$ :

$$
\begin{gathered}
f_{n+1}=f_{n} A_{n}+B_{n}, \quad A_{n}=A_{n}(n), B_{n}=0, \\
f_{1} \equiv W, \quad F_{N} \equiv f_{1}+f_{2}+\ldots+f_{N} \quad \text { for } \quad \mathrm{n}=1,2,3, \ldots, \mathrm{N} . ; N=1,2, \ldots, \infty .
\end{gathered}
$$

For mentioned assumptions and boundary condition, the recurrent formula (1) has the following particular solution describing the wear values during $n$-th time unit of operation:

$$
f_{n}=\frac{W}{A_{n}} \cdot \prod_{j=1}^{n} A_{j} \quad \text { for } \quad n=1,2,3, \ldots, N ; \quad N=1,2, \ldots, \infty .
$$

Expanding formula (3) for $\mathrm{n}=1,2, \ldots, \mathrm{N}$, we obtain the wear values in following particular time units:

$$
f_{1}=W, \quad f_{2}=W A_{1}, \quad f_{3}=W A_{1} A_{2}, \ldots, f_{N}=W A_{1} A_{2} \ldots \ldots . . A_{N-1} .
$$

Thus from (4) we obtain the wear during infinite time unit in following form:

$$
f_{\infty}=\frac{W}{A_{\infty}} \cdot \prod_{j=1}^{\infty} A_{j} .
$$

Cumulative wear after $\mathrm{N}$ and after infinite time units has the following form:

$$
F_{N}=\sum_{n=1}^{N} f_{n}=W \sum_{n=1}^{N}\left(\frac{1}{A_{n}} \cdot \prod_{j=1}^{n} A_{j}\right), \quad F_{\infty}=\sum_{n=1}^{\infty} f_{n}=W \sum_{n=1}^{\infty}\left(\frac{1}{A_{n}} \cdot \prod_{j=1}^{n} A_{j}\right), \mathrm{N}=1,2, \ldots, \infty .
$$




\section{Example 1}

Determine the wear cumulative function if variable dimensionless coefficient obtained from experimental measurements has the following form $[3,13,14]$ :

$$
\begin{aligned}
& A_{n}=1+\frac{1}{n} \quad \text { for } n=1,2,3, \ldots \quad \text { whereas } \\
& A_{1} \cdot A_{2} \cdot A_{3} \cdot \ldots . . A_{n}=\left(1+\frac{1}{1}\right) \cdot\left(1+\frac{1}{2}\right) \cdot\left(1+\frac{1}{3}\right) \ldots\left(1+\frac{1}{n}\right)=\frac{2}{1} \cdot \frac{3}{2} \cdot \frac{4}{3} \cdot \frac{5}{4} \ldots\left(\frac{n}{n-1}\right)\left(\frac{n+1}{n}\right)=n+1 .
\end{aligned}
$$

Parameter (7) describes the partial product where differences of variable wear parameter values $A_{n}$ between successive time units are decreasing accordingly with harmonic sequence $1 / \mathrm{n}$. By virtue $(3),(7)$, during finite $\mathrm{n}$ and infinite steps $(\mathrm{n} \rightarrow \infty)$ we obtain following wear values:

$$
\begin{aligned}
& f_{n}=\frac{W}{1+\frac{1}{n}} \cdot \prod_{j=1}^{n}\left(1+\frac{1}{n}\right)=\frac{W n(n+1)}{n+1}=W n \quad \text { for } n=1,2,3, \ldots, N ; \quad N=1,2, \ldots, \infty, \\
& f_{\infty}=W \prod_{j=1}^{\infty}\left(1+\frac{1}{n}\right)=\lim _{n \rightarrow \infty} W n=\infty .
\end{aligned}
$$

From (8) follows, that that presented wear values sequence is divergent. By virtue (6), (7), the cumulative wear (i.e. sum of wear values) sequence has following form:

$$
F_{N}=\sum_{n=1}^{N} f_{n}=W \sum_{n=1}^{N}\left(\frac{n}{n+1} \cdot \prod_{j=1}^{n} \frac{n+1}{n}\right), \quad \mathrm{N}=1,2, \ldots, \infty, \quad F_{\infty}=\sum_{n=1}^{\infty} f_{n}=W \sum_{n=1}^{\infty}\left(\frac{n}{n+1} \cdot \prod_{j=1}^{n} \frac{n+1}{n}\right)=\infty
$$

Cumulative sum of wear values sequence is divergent.

\section{Second case of wear cumulative process identifications}

In this intersection for second case we assume constant dimensionless coefficients $A_{n}$, and variable dimensional free term $\mathrm{B}_{\mathrm{n}}$. Thus we consider the following particular case of wear recurrence and cumulative wear equation $[3,13,14]$ :

$$
\begin{aligned}
& f_{n+1}=f_{n} A_{n}+B_{n}, \quad A_{n}=A=\text { const. }, B_{n}=B_{n}(n), \\
& f_{1} \equiv W, \quad F_{N} \equiv f_{1}+f_{2}+\ldots+f_{N} \quad \text { for } \quad \mathrm{n}=1,2,3, \ldots, \mathrm{N} ; N=1,2, \ldots, \infty .
\end{aligned}
$$

For mentioned assumption and boundary condition (10), the recurrent formula (1) has the following particular solution describing the wear values during $n$-th time unit of operation [13, 14]:

$$
f_{n}=\frac{W}{A} \cdot A^{n}-\frac{B_{n}}{A}+\frac{A^{n}}{A} \sum_{k=1}^{n}\left(\frac{B_{k}}{A^{k}}\right) \quad \text { for } \quad n=1,2,3, \ldots, N ; \quad N=1,2, \ldots, \infty .
$$

Expanding formula (11) for $n=1,2, \ldots, N$, we obtain the wear values in following particular time units:

$$
\begin{aligned}
& f_{1}=W, \quad f_{2}=W A+B_{1}, \quad f_{3}=W A^{2}+B_{1} A+B_{2}, \\
& f_{N}=W A^{n-1}+B_{1} A^{n-2}+B_{2} A^{n-3}+\ldots+B_{n-2} A+B_{N-1} .
\end{aligned}
$$

Hence, from (12) we obtain the wear during infinite time units in the following form: 


$$
f_{\infty}=\frac{W}{A} \cdot A^{\infty}-\frac{B_{\infty}}{A}+\frac{A^{\infty}}{A} \sum_{k=1}^{\infty}\left(\frac{B_{k}}{A^{k}}\right) .
$$

By virtue (11), the cumulative wear after $\mathrm{N}$ and infinite time units has the following form:

$$
\begin{aligned}
& F_{N}=\sum_{n=1}^{N} f_{n}=\frac{W}{A} \cdot \sum_{n=1}^{N} A^{n}-\frac{1}{A} \sum_{n=1}^{N} B_{n}+\frac{1}{A} \sum_{n=1}^{N}\left[A^{n} \sum_{k=1}^{n}\left(\frac{B_{k}}{A^{k}}\right)\right], \quad N=1,2 .,,, . \infty, \\
& F_{\infty}=\sum_{n=1}^{\infty} f_{n}=\frac{W}{A} \cdot \sum_{n=1}^{\infty} A^{n}-\frac{1}{A} \sum_{n=1}^{\infty} B_{n}+\frac{1}{A} \sum_{n=1}^{\infty}\left[A^{n} \sum_{k=1}^{n}\left(\frac{B_{k}}{A^{k}}\right)\right] .
\end{aligned}
$$

Example 2

Determine the wear cumulative function if constant dimensionless coefficient A and variable dimensional free term obtained from experimental measurements have the following form:

$$
A=\frac{1}{2}=\text { const }, \quad B_{n}=b n, \quad b-\text { const. }
$$

In this case the dimensional wear values $f_{n+1}$ in the next time units step equal to sum $A f_{n}+B_{n}$ of dimensional wear $f_{n}$ in foregoing step multiplied by constant dimensionless factor $A=1 / 2$ plus some linear increasing factor $\mathrm{b} \cdot \mathrm{n}$ for $\mathrm{b}$ dimensional arbitrary constant.

By virtue of (11), (15) during finite $\mathrm{n}$ steps of considered wear sequence, we obtain following wear values:

$$
f_{n}=2 W \cdot\left(\frac{1}{2}\right)^{n}-2 b \cdot n+2 b\left(\frac{1}{2}\right)^{n} \sum_{k=1}^{n}\left(k \cdot 2^{k}\right) \quad \text { for } \quad n=1,2,3, \ldots, N ; \quad N=1,2, \ldots, \infty .
$$

Taking into account UOS operator of summation, we obtain [13]:

$$
\sum_{k=1}^{n}\left(k \cdot 2^{k}\right)=2+2 \cdot 2^{n} \cdot(n-1) \quad \text { for } \quad n=1,2,3, \ldots, N ; \quad N=1,2, \ldots, \infty .
$$
form:

Putting (17) into (16) and after terms ordering, we obtain wear values (16) in the following

$$
\begin{gathered}
f_{n}=2 W \cdot\left(\frac{1}{2}\right)^{n}-2 b \cdot n+4 b(n-1)+4 b\left(\frac{1}{2}\right)^{n}=2 W \cdot\left(\frac{1}{2}\right)^{n}+2 b \cdot n-4 b+\frac{4 b}{2^{n}} \\
\text { for } n=1,2,3, \ldots, N ; \quad N=1,2, \ldots, \infty .
\end{gathered}
$$

During infinite step (for $n \rightarrow \infty$ ) of considered wear sequence (18), the wear value tends to infinity:

$$
f_{\infty}=-4 b+2 b \cdot \lim _{n \rightarrow \infty} n=+\infty .
$$

By virtue (18), the cumulative wear i.e. the sum of wear values, after $\mathrm{N}$ time units have following form:

$$
F_{N}=\sum_{n=1}^{N} f_{n}=2 W \cdot \sum_{n=1}^{N}\left(\frac{1}{2}\right)^{n}+2 b \cdot \frac{N(N+1)}{2}-4 b N+4 b \sum_{n=1}^{N}\left(\frac{1}{2}\right)^{n}, \text { for } n=1,2,3, \ldots, N ; \quad N=1,2, \ldots, \infty .(20)
$$

Now we apply following sum of geometrical convergent series:

$$
\sum_{n=1}^{N}\left(\frac{1}{2}\right)^{n}=\frac{1}{2}\left(\frac{1-(0.5)^{N}}{1-0.5}\right) \text { for } \quad n=1,2,3, \ldots, N ; \quad N=1,2, \ldots, \infty
$$

We put (21) into (20) hence cumulative wear function tends to the following final form: 


$$
F_{N}=\sum_{n=1}^{N} f_{n}=2(W+2 b) \cdot\left[1-\left(\frac{1}{2}\right)^{N}\right]+b \cdot\left(N^{2}-3 N\right), \text { for } \quad n=1,2,3, \ldots, N ; \quad N=1,2, \ldots, \infty
$$

After infinite steps $(\mathrm{N} \rightarrow \infty)$ of considered wear process $(22)$, we obtain after infinite time units the following cumulative sum of wear values:

$$
F_{\infty}=\sum_{n=1}^{\infty} f_{n}=2(W+2 b)+b \cdot \lim _{N \rightarrow \infty}\left(N^{2}-3 N\right)=+\infty \quad \text { for } \quad n=1,2,3, \ldots, N ; \quad N=1,2, \ldots, \infty .
$$

Cumulative sum of wear values is divergent.

\section{Third case of wear cumulative process identifications}

In this intersection, we assume constant dimensionless coefficients A and constant dimensional free term B in recurrent wear equation. Hence, we consider the following simple particular case of wear recurrence and cumulative wear equation $[3,13,14]$ :

$$
\begin{aligned}
& f_{n+1}=f_{n} A_{n}+B_{n}, \quad A_{n}=A=\text { const }, \quad B_{n}=B=\text { const }, \\
& f_{1} \equiv W, \quad F_{N} \equiv f_{1}+f_{2}+\ldots+f_{N} \quad \text { for } \quad \mathrm{n}=1,2,3, \ldots, \mathrm{N} . ; N=1,2, \ldots, \infty .
\end{aligned}
$$

For mentioned assumptions and boundary condition (24), the recurrent formula (1) has the following particular solution describing the wear values during $n$-th time unit of operation:

$$
f_{n}=\frac{W}{A} \cdot A^{n}-\frac{B}{A}+\frac{B}{A} A^{n} \sum_{k=1}^{n}\left(\frac{1}{A^{k}}\right) \quad \text { for } \quad n=1,2,3, \ldots, N ; \quad N=1,2, \ldots, \infty .
$$

Expanding formula (25) for $n=1,2, \ldots, N$, we obtain the wear values in following particular time units:

$$
f_{1}=W, \quad f_{2}=W A+B, \quad f_{3}=W A^{2}+B A+B, \quad \ldots, \quad f_{N}=W A^{n-1}+B A^{n-2}+\mathbf{B} A^{n-3}+\ldots+B A+B .
$$

Taking into account the sum of geometrical series, and after terms ordering, solution (25) for $\mathrm{A}>0$ has the form:

$$
f_{n}=W A^{n-1}+B \frac{A^{n-1}-1}{A-1} \quad \text { for } \quad n=1,2,3, \ldots, N ; \quad N=1,2, \ldots, \infty .
$$

For $A \equiv 1$ wear solution (27) and its value in infinity operation time units tend to the form:

$$
\begin{gathered}
f_{n}=\lim _{A \rightarrow 1}\left(W A^{n-1}+B \frac{A^{n-1}-1}{A-1}\right)=W+B \cdot(n-1), \text { for } n=1,2,3, \ldots, N ; \quad N=1,2, \ldots, \infty . \\
f_{\infty}=\lim _{n \rightarrow \infty} f_{n}=W+\infty=\infty .
\end{gathered}
$$

For $A>0$ and $A \neq 1$ wear solution (27) and its value in infinity operation time units have the form:

$$
\begin{gathered}
f_{n}=\left(W-\frac{B}{1-A}\right) A^{n-1}+\frac{B}{1-A} \text { for } n=1,2,3, \ldots, N ; \quad N=1,2, \ldots, \infty . \\
f_{\infty}=\lim _{\substack{n \rightarrow \infty \\
A>1}} f_{n}=\frac{B}{1-A}+\infty=\infty ;
\end{gathered}
$$




$$
f_{\infty}=\lim _{\substack{n \rightarrow \infty \\ 0<A<1}} f_{n}=\frac{B}{1-A} .
$$

For $\mathrm{A} \equiv 1$ cumulative wear after $\mathrm{N}$ time units of operation i.e. finite and infinite sum for $\mathrm{n}=1,2$, $\ldots, \mathrm{N}$ of function $(28 \mathrm{~b})$ tends to the following final form:

$$
\begin{aligned}
F_{N} & =\sum_{n=1}^{N} f_{n}=\sum_{n=1}^{N}[W+B \cdot(n-1)]=W N+\frac{B N(N-1)}{2}, \quad N=1,2, \ldots, \infty, \\
F_{\infty} & =\lim _{\substack{N \rightarrow \infty \\
B>0}} F_{N}=\infty+\infty=\infty .
\end{aligned}
$$

The cumulative sum of wear values after infinite $(\mathrm{N} \rightarrow \infty)$ time units is divergent. For $\mathrm{A} \neq 1$ cumulative wear after $\mathrm{N}$ time units of operation i.e. finite and infinite sum for $\mathrm{n}=1,2, \ldots, \mathrm{N}$ of function $(29 a)$ tends to the following final forms:

$$
\begin{gathered}
F_{N}=\sum_{n=1}^{N} f_{n}=\left(W-\frac{B}{1-A}\right) \sum_{n=1}^{N} A^{n-1}+\sum_{n=1}^{N}\left(\frac{B}{1-A}\right)= \\
=\left(W-\frac{B}{1-A}\right) \frac{A^{N}-1}{A-1}+\frac{B N}{1-A}=W \cdot \frac{1-A^{N}}{1-A}+\frac{B}{1-A}\left(N-\frac{1-A^{N}}{1-A}\right) \quad \text { for } \quad N=1,2, \ldots, \infty .
\end{gathered}
$$

For each $\mathrm{N}$ and for $\mathrm{B}>0, \mathrm{~A}>1$ or for $\mathrm{B}>0,0<\mathrm{A}<1$ following inequalities and limits are true [13]:

$$
\begin{gathered}
\frac{B}{1-A}\left(N-\frac{1-A^{N}}{1-A}\right)>0, \quad \frac{1-A^{N}}{1-A}>0, \frac{B}{1-A}\left(N-\frac{1-A^{N}}{1-A}\right) \stackrel{A>1,0<A<1}{\longrightarrow} \infty, \\
\frac{1-A^{N}}{1-A} \stackrel{A>1}{\longrightarrow} \infty, \quad \frac{1-A^{N}}{1-A} \stackrel{0<A<1}{\longrightarrow} \frac{1}{1-A} .
\end{gathered}
$$

Hence:

$$
F_{\infty}=\lim _{\substack{N \rightarrow \infty \\ 0<A<1}} F_{N}=\frac{W}{1-A}+\infty=\infty ; \quad F_{\infty}=\lim _{\substack{N \rightarrow \infty \\ A>1}} F_{N}=\infty+\infty=\infty .
$$

After infinite $(\mathrm{N} \rightarrow \infty)$ time units, the cumulative sum of wear values is divergent in both abovementioned cases.

\section{Example 3}

After first time units, the considered slide friction nod attains wear values $\mathrm{W} \mathrm{mm}^{3}$. Determine sum of the wear after ten time units if we know that for sliding nod we have following parameters obtained in experimental way: $A=0.25, B=0.05 \mathrm{~mm}^{3}$. After measurements we obtain wear (during 10 days) after first time unit $W=0.1 \mathrm{~mm}^{3}$. Determine wear after 100 days i.e. after $N=10$ time units. Solutions:

Putting the values $\mathrm{W}, \mathrm{A}, \mathrm{B} \mathrm{N}$ into the formula (31) we obtain the following dimensional wear value after 10 time units: 


$$
\begin{gathered}
F_{10}=\sum_{n=1}^{10} f_{n}=W \cdot \frac{1-A^{10}}{1-A}+\frac{B}{1-A}\left(10-\frac{1-A^{10}}{1-A}\right)= \\
=0.1 \mathrm{~mm}^{3} \frac{1-(0.25)^{10}}{1-0.25}+\frac{0.05 \mathrm{~mm}^{3}}{1-0.25}\left[10-\frac{1-(0.25)^{10}}{1-0.25}\right]=0.7111110687 \mathrm{~mm}^{3} .
\end{gathered}
$$

The cumulative sum of ear values after ten $(\mathrm{N}-10)$ time units attains value $0.7111110687 \mathrm{~mm}^{3}$.

\section{Conclusions}

1. This paper presents the influence of material properties of human joints, slide journal bearing, cams, piston rings, tires, brakes, dirt seals, belts, shoes, fabrics, electrical contacts, tape and CD reader heads, on the values of the wear cumulative process presenting the sum of wear in the succeeding time periods of the operation.

2. The application of the presented theory may be need in the control methods for the determination of cumulative wear values i.e. sum of wear after arbitrary finite and infinite time units of operation.

3. The examples presented in this paper examine the convergences and divergences of the wear cumulative values after finite and infinite time units of operation.

4. The theory presented in this paper indicates the influence of variable coefficients which are determined from the experiments on the cumulative wear values and its convergences speed after many time units of operation.

\section{References}

[1] Bharat, Bhushan, Handbook of Micro/Nano Tribology, second ed. CRC Press, Boca Raton, London, New York, Washington D.C., 1999.

[2] Bharat, Bhushan, Nanotribology and nanomechanics of MEMS/NEMS and BioMEMS/ BioNEMS materials and devices, Microelectronic Engineering, 84, pp. 387-412, 2007.

[3] Chizhik, S., Wierzcholski, K., Trushko, A., Zbytkova, M., Miszczak, A., Properties of Cartilage on Micro- and Nanolevel, Advances in Tribology, Vol. 2010, doi:10.1155/2010/243150, 2010

[4] Hedagehatte, V., Hilgert, J., Kraft, O., Huber, N., Multi time scale simulation for wear prediction in micro-gears, Wear, 268, pp. 316-324, 2010.

[5] Hedagehatte, V., Huber, N., Kraft, O., Finite Element Based Simulation of Dry Sliding Wear, Modelling and Simulation in Materials Science and Engineering, 13, pp. 57-75, 2004.

[6] Jang, G. H., Seo, C. H., Ho Scong Lee, Finite element model analysis of an HDD considering the flexibility of spinning disc-spindle, head-suspension-actuator and supporting structure. Microsystem Technologies, 13, pp. 837-847, 2007.

[7] Kim, K. T., Ludema, K. C., Low cycle fatigue as an initiating mechanism of scuffing, ASME, Journal of Tribology, 117, 231, 1995.

[8] Ludema, K. C., Friction, Wear, Lubrication, CRC Press, N.Y., London, Tokyo 1996.

[9] Miu, D. K., Tai, Y. C., Silicon Micromachined Scaled Technology, IEEE Trans. Ind. Electron., 42, pp. 234-239, 1995.

[10] Mastrangelo, C. H., Adhesion Related Failure Mechanisms in Micromechanical Devices, Tribology Letter, 3, pp. 233-238, 1997.

[11] Rezaei Ali, Wim Van Paepegem, De Baest Patrick, Wouter Ost, Degrieck Joris, Adaptive finite element simulation of wear evolution in radial sliding bearings, Wear, 296, pp. 660-671, 2012.

[12] Venkatesan, S., Bharat Bhushan, The sliding friction and wear behavior of single-cristal, Polycristalline and oxidized silicon, Wear, 171, pp. 25-32, 1994.

[13] Wierzcholski, K., Unified summation equations and their applications in tribology wear process, Bulletin of the Polish Academy of Sci, Technical Sci., Vol. 61, No. 1, pp. 1-21, 2013. 
[14] Wierzcholski, K., Solution of recurrences with variable coefficients for slide bearing wear determination, Journal of KONES Powertrain and Transport, Vol. 20, No. 3, pp. 427-433, 2013.

[15] Yang, L. J., A test methodology for the determination of wear coefficient, Wear, 259, pp. 1453-1461, 2005. 